\title{
BER PERFORMANCE OF OFDM WITH DISCRETE WAVELET TRANSFORM FOR TIME DISPERSIVE CHANNEL
}

\author{
Kavita Trivedi $^{1}$, Anshu Khare ${ }^{2}$, Saurabh Dixit ${ }^{3}$ \\ ${ }^{1}$ Department of Electronics and communication, Lucknow, India \\ ${ }^{2}$ Department of Electronics and communication, Lucknow, India \\ ${ }^{3}$ Department of Electronics and communication, Lucknow, India
}

\begin{abstract}
This paper presents a model which is discrete wavelet transform based (orthogonal frequency division multiplexing) OFDM system for next generation wireless communication. Fourier transform based OFDM system signals only overlap in the frequency domain while the wavelet transform signals overlap both in the time and frequency domain so there is no need of the cyclic prefix $(C P)$ in the proposed model. There are different types of wavelet transform are available the performance of the wavelet based OFDM system using modulation QAM was assessed by various parameters such as Bit error rate and constellation diagram. In OFDM inter symbol interference and the inter carrier interference exist. Due to the multipath wireless channel. wavelet based transmission is investigated and its shown that this technique is better at suppressing ISI and ICI than conventional OFDM. the results presented in this paper are based on computer simulation performed using MATLAB software.
\end{abstract}

Keywords-DWT, OFDM, BER, AWGN

\section{INTRODUCTION}

Wavelet represent a successful story of the last decades in signal processing application. Thus these signals, with some remarkable properties, are widely used in various domain as compression, denoising segmentation by the other hand in data communication the some successful story can be assigned to multi carrier modulation on techniques .most of the data transmission systems now a days use orthogonal frequency division multiplexing (OFDM).we can mention here WiFi(IEEE 802.11),WiMAX(IEEE 802.16).It is especially the very good resilience of OFDM.OFDM system is a multicarrier system which process signals to be transmitted in parallel at different frequencies simultaneously. the system employs quad intervals or cyclic prefix (CP) so that the delay spread of the channel becomes longer than the channel impulse response. many researchers have investigated the use of wavelet based to replace fourier based OFDM and found out that the wavelet based has more advantages than fourier based OFDM because in OFDM some drawbacks can be counteracted, thus the side lobes of the OFDM spectrum contain an important amount of energy causing interference in the adjacent bands. in the orthogonality of the wavelet carrier relies on both their time position and their frequency .this make the transmission less sensitive to doppler and leading to noticeable BER improvements.

In the next section we will discuss the fourier based OFDM, in section 3 wavelet based OFDM, in section 4 simulation results are presented and commented last section is dedicated to some conclusions.

\section{FOURIER-BASED OFDM}

The data $\left\{d_{k}\right\}$ is processed by modulation.to map the data before IFFT , with $\mathrm{N}$ sub carriers its output is the sum of the information signals in the discrete time bearing as following-

$$
X_{K}=\frac{1}{\sqrt{N}} \sum_{m=0}^{N-1} X_{m} e^{j 2 \pi k m / N}
$$

Where $\left.X_{k} / 0 \leq \mathrm{k} \leq \mathrm{N}-1\right\}$ is a sequence in discrete time domain $\left\{X_{m} / 0 \leq \mathrm{m} \leq \mathrm{N}-1\right\}$ are complex numbers in discrete frequency domain the cyclic prefix (CP)is added before transmission to minimize the inter symbol interference at the receiver side the process is reversed to obtain and decoded the data the $\mathrm{CP}$ is removed to obtain the data in discrete time domain. The output of FFT is the sum of the received signal in discrete frequency domain as follows-

$$
X_{m}=\sum_{k=0}^{N-1} X_{k} e^{-j 2 \pi k m / N}
$$

\section{WAVELET BASED OFDM}

The orthogonals sets of codes are functional for wavelet signalling and that one orthogonality remained broken over both time as well as scaling. the analysis of DWT signals through different resolution next to the different frequency through using the decomposition signals into an estimate comprising abrasive then in depth data the ripple function are denoted by low pass as well as high pass filters the low pass filters is denoted by $\mathrm{r}[\mathrm{n}]$.the high pass filter is denoted by $s[n]$.the high pass filters allow all high frequency signal, the low pass filters allow below the highest frequency signal, the filter makes the determination half and also the 
mounting factor vestiges same the splitting of filters decomposition, reconstruction as shown in figure 1,2 and 3 to avoid redundant samples using down sampling by factor 2 . The decomposition of the signal is expressed as-

$$
\begin{gathered}
Z_{H I G H}[\mathrm{k}]=\sum_{n=0}^{\infty} x[n] g[2 k-n] \\
Z_{L O W}[\mathrm{k}]=\sum_{n=0}^{\infty} x[n] h[2 k-n]
\end{gathered}
$$

The time resolution is reduced by decomposition method by using the factor by 2

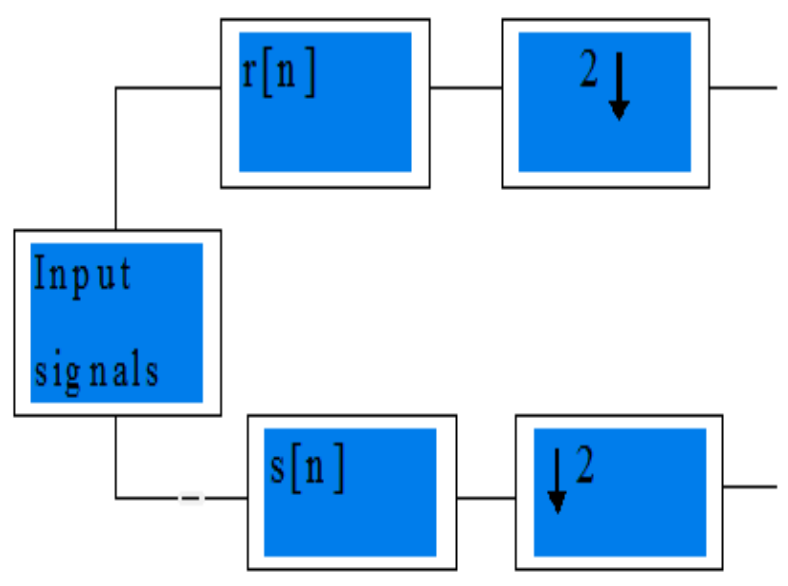

Fig -1 splitting of filters

IFFT plus FFT remained prepared through wavelet transforms DWT and IDWT. by way of this transform it does not use CP ,so the spectral suppression of the channels was improved the scaling constant is occausnaly denoted approximated coefficients the good ripple then creates as a well meaning choice aimed at many application ,images, synthesis, nuclear engineering, music, data compression, computer graphics and animations, human vision ,radar astronomy, biomedical engineering, magnetic resonance.

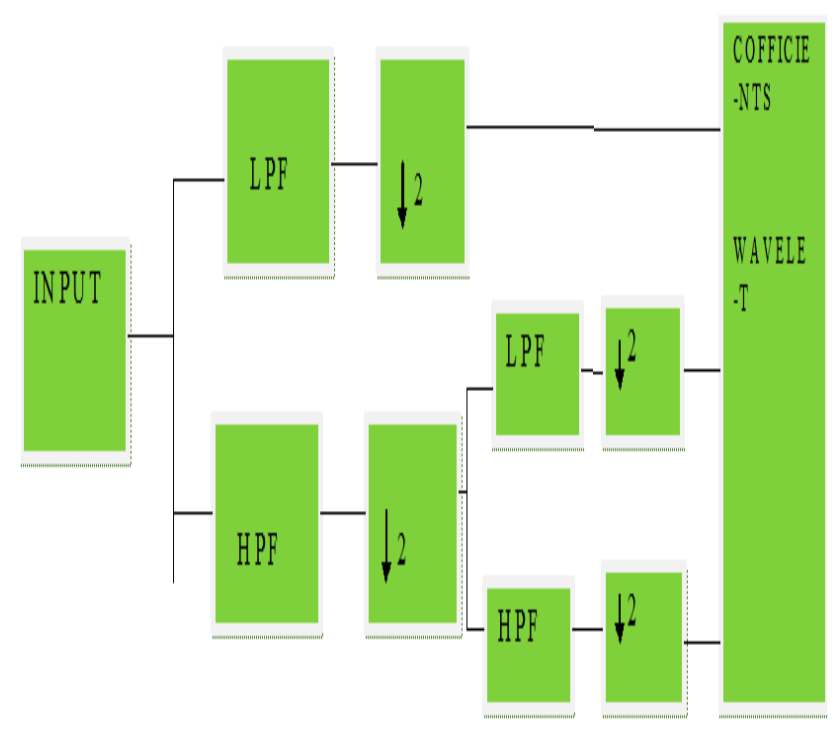

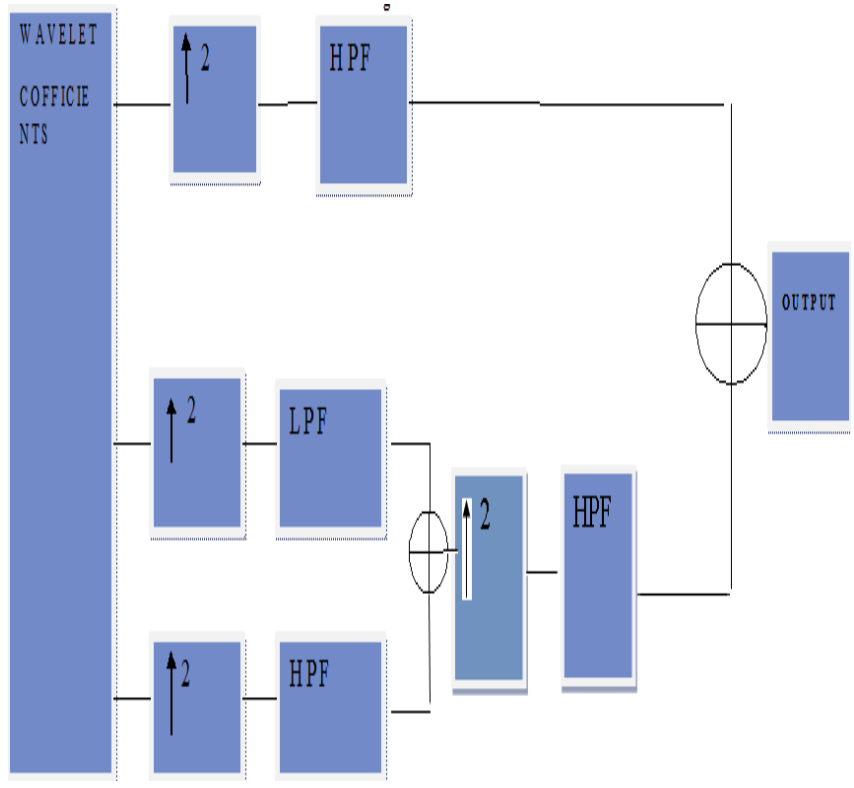

Fig 3 Diagram for DWT transform

\section{SIMULATION AND RESULTS}

Two transform methods FFT based OFDM system and DWT based OFDM systems are implemented using MATLAB and graphical results are found showing the bit error rate probabilities of the systems, the parameters which are used to simulate these both proposed systems are shown in table 1 .

Table1 parameters selection for simulation of FFT based

\begin{tabular}{|l|l|l|}
\hline \multicolumn{3}{|c|}{ OFDM and DWT } \\
\hline $\begin{array}{l}\text { Parameters of data } \\
\text { subcarriers }\end{array}$ & $\mathbf{5 2}$ & $\mathbf{5 2}$ \\
\hline Cyclic prefix & $\begin{array}{l}\text { 1/4 of data sub } \\
\text { carriers }\end{array}$ & $\mathbf{0}$ \\
\hline FFT size & $\mathbf{6 4}$ & - \\
\hline wavelet & - & Haar,db1 \\
\hline $\begin{array}{l}\text { Modulation } \\
\text { method }\end{array}$ & QAM,BPSK & BPSK \\
\hline channel & AWGN & AWGN \\
\hline $\begin{array}{l}\text { Total no of } \\
\text { symbols }\end{array}$ & $\mathbf{1 0} \mathbf{4}$ & $\mathbf{1 0} \mathbf{4}$ \\
\hline Pilot insertion & $\mathbf{8}$ & - \\
\hline
\end{tabular}

In this section BER performance achieved using the MATLAB simulation of different modulation schemes.I

\subsection{Power Spectrum of FFT Based OFDM Systems}

In the FFT based OFDM system the power spectrum having a major fading effect and it reduce the system performance

Fig 2-Diagram for IDWT transforms 


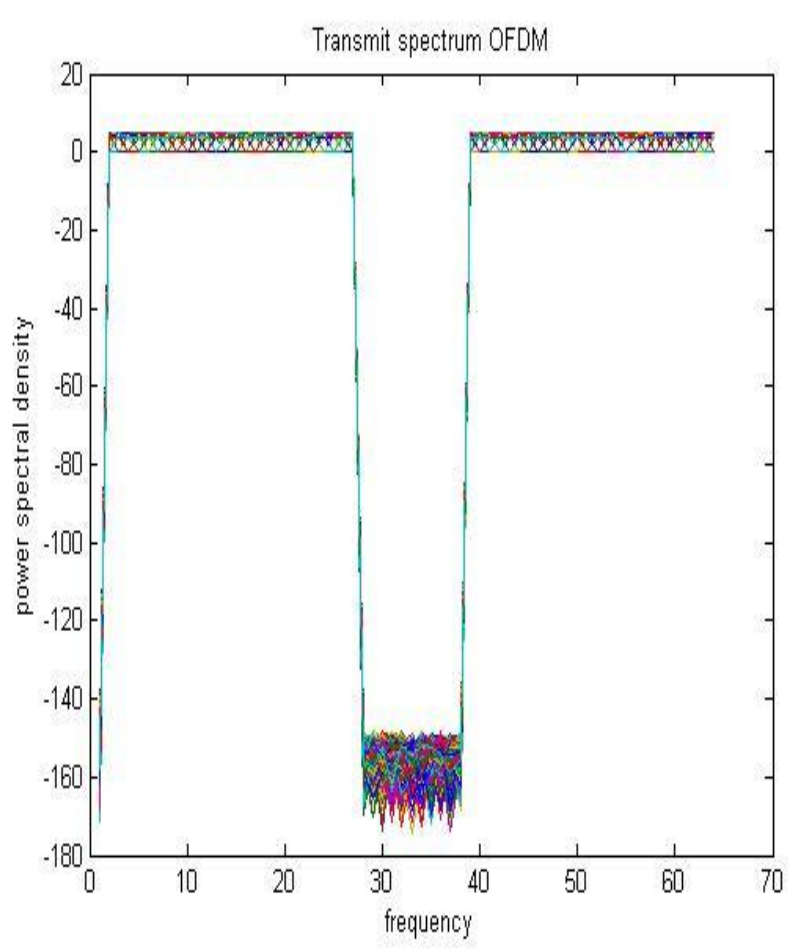

Fig 4 power spectrum of OFDM system

\subsection{Power spectrum of DWT based OFDM System}

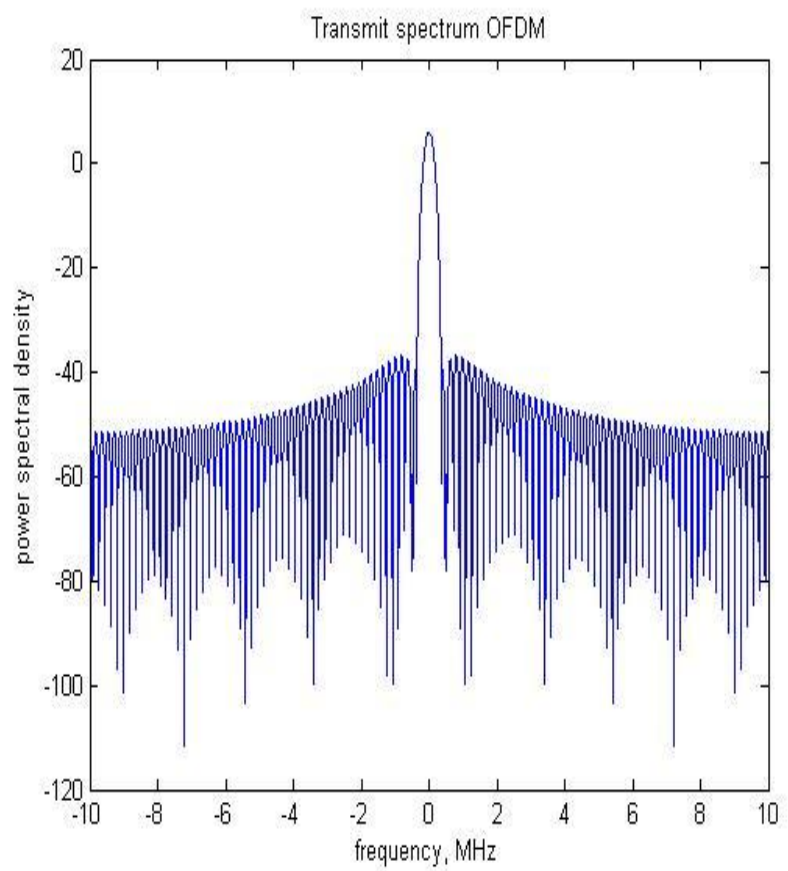

Fig 5 Power spectrum of DWT based OFDM

In DWT based OFDM system power spectrum using very less bandwidth .so most of bandwidth saved .as campare the spectrum of FFT -OFDM and DWT OFDM wavelet based OFDM increasing the system performance.

\subsection{Comparison of FFT based OFDM with DWT - OFDM System over the AWGN Channel}

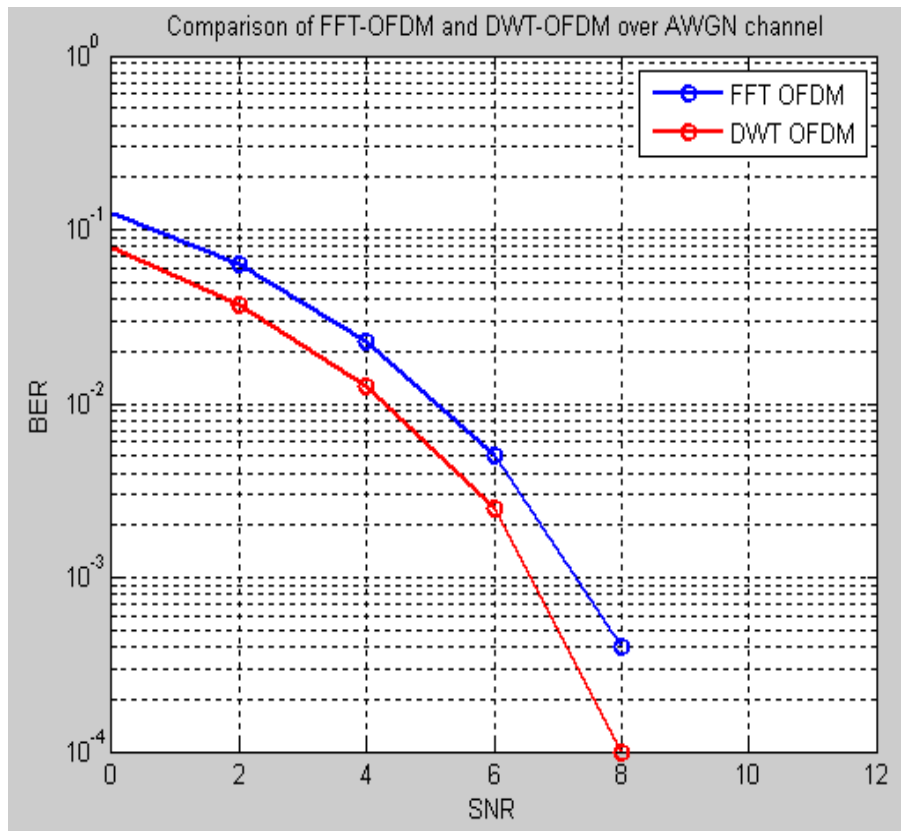

Fig-6 BER BPSK over AWGN channel

The following fig3 BPSK over AWGN channel indicates that a SNR $=4 \mathrm{~dB}$ the BER performance is $10^{\wedge}-2$ and at SNR $=8 \mathrm{~dB}$ the BER performance isnearly equal to $10^{\wedge}-4$ in DWT OFDM system which is less than FFT -OFDM system.

\section{CONCLUSIONS}

The OFDM system implemented with wavelet transform gives better spectrum efficiency.the main disadvantages of the conventional FFT based OFDM system is the addition of cyclic prefix to remove the ISI and ICI.in DWT based OFDM system,IFFT and FFT have been replaced by IDWT and DWT wavelet transform maintain the orthogonality.so no need to add cyclic prefix in DWT based OFDM system.

The BER performance of the FFT based OFDM systems can be found over AWGN channel using the BPSK modulation .from the plots of the BER as a function of the signal to noise ratio(SNR), it can be concluded that when the signals to noise ration(SNR) is very low and the does not have any impact on the BER but if the SNR is vry high it highly effect the BER performance $n$ DWT based OFDM system the performance of BER is less effected by the SNR but in the FFT based OFDM the performance highly effected .so it concluded that DWT performance better than the FFT based OFDM system.

\section{REFERENCES}

[1] Meenakshi,S.Prabha,N.R.RaajaEVALUATION OF BER FOR VARIOUSFADING CHANNEL IN DWT BASED MIMO-OFDM SYSTEMD." Meenakshi et al. / International Journal of Engineering and Technology (IJET) 
[2] You-Seok Lee, Hyun-Chool Shin, and Hyoung-Nam Kim, Member, IEEE"'Channel Estimation Based on a Time-Domain Threshold for OFDM Systems"IEEE TRANSACTIONS ON BROADCASTING, VOL. 55, NO. 3, SEPTEMBER 2009

[3] MARIUS OLTEANOn the Wavelet OFDM Performance in Time Variant Channels: Choosing the Number of DWT Iterations

[4] Vijay Kumar Kol and Agya MishraDiscrete Wavelet Transform based OFDM-IDMA System with AWGN Channel978-1-4673-5630-5//13/\$31.00 @2013 IEEE

[5] Khaizuran Abdullah and Zahir M. Hussain, SMIEEEPerformance of Fourier-Based and WaveletBased OFDM for DVB-T Systems2007 Australasian Telecommunication Networks and Applications Conference December 2nd - 5th 2007, Christchurch, New Zealand.

[6] Khaizuran Abdullah, * Saidatul Izyanie Kamarudin, * Nadiatul Fatiha Hussin, Sigit PW Jarrot and Ahmad Fadzil IsmailImpulsive Noise Effects on DWTOFDM versus FFT-OFDM2011 17th Asia-Pacific Conference on Communications (APCC).

[7] Gerald Matz and Franz Hlawatsch Fundamentals of Time-Varying Communication Channels in Wireless Communications over Rapidly Time-Varying Channels eds. Franz Hlawatsch and Gerald Matz Amsterdam, The Netherlands: Academic Press Chapter 1, pp. 1-63

[8] Hilal A. Fadhila, Hassan YousifAhmedb, S. A. Aljunida Performance analysis of Hybrid optical OFDM system with high order dispersion compensation 2011 17th Asia-Pacific Conference on Communications (APCC) 2nd - 5th October 2011 | Sutera Harbour Resort, Kota Kinabalu, Sabah, Malaysia

[9] N.R.Raajan, B.Monisha, M.Ram Kumar, A.Jenifer Philomina, M.V.Priya, D.Parthiban, S.Suganya Design and Implementation of Orthogonal Wavelet

[10] Division Multiplexing (OHWDM) with Minimum Bit Error Rate 978-1-4673-0132-91111\$26.00@2011 IEEE

[11] Volkan Kumbasar' and Oguz Kucur' Searching Better Wavelet Packet Tree for 1SI and leI Reduction in WOFDM 1-4244-2589-1/09/\$20.00 @2009 IEEE 